\title{
Synthesis and Characterization of Graphene Oxide and Reduced Graphene Oxide Thin Films Deposited by Spray Pyrolysis Method
}

\author{
M. S. Eluyemi ${ }^{1}$, M. A. Eleruja ${ }^{1}$, A. V. Adedeji ${ }^{2}$, B. Olofinjana ${ }^{1 *}$, 0. Fasakin'1, 0. O. Akinwunmi ${ }^{1}$, \\ 0. O. Ilori ${ }^{3}$, A. T. Famojuro" ${ }^{4}$ S. A. Ayinde', E. O. B. Ajayi ${ }^{1}$ \\ ${ }^{1}$ Department of Physics and Engineering Physics, Obafemi Awolowo University, Ile-Ife, Nigeria \\ ${ }^{2}$ Department of Chemistry and Physics, Elizabeth City State University, Elizabeth City, NC, USA \\ ${ }^{3}$ Department of Electronics and Electrical Engineering, Obafemi Awolowo University, Ile-Ife, Nigeria \\ ${ }^{4}$ Department of Chemistry, Obafemi Awolowo University, Ile-Ife, Nigeria \\ Email: *olofinb@oauife.edu.ng
}

Received 20 April 2016; accepted 26 July 2016; published 29 July 2016

Copyright (C) 2016 by authors and Scientific Research Publishing Inc.

This work is licensed under the Creative Commons Attribution International License (CC BY).

http://creativecommons.org/licenses/by/4.0/

(c) (i) Open Access

\section{Abstract}

Graphene Oxide (GO) was chemically synthesized from Natural Flake Graphite (NFG). The GO was chemically reduced to Reduced Graphene Oxide (RGO) using hydrazine monohydrate. Thin films of GO and RGO were also deposited on sodalime glass substrate using spray pyrolysis technique (SPT). The samples were characterized using Fourier Transform Infrared (FTIR) spectroscopy, Scanning Electron Microscopy (SEM) with Energy Dispersive X-Ray (EDS) facility attached to it, UV-Visible Spectrometry and Four-Point probe. The FTIR spectra showed the addition of oxygen functionality groups in GO while such groups was drastically reduced in RGO. SEM micrograph of GO thin film showed a porous sponge-like structure while the micrograph of RGO thin film showed evenly distributed and well connected graphene structure. The EDX spectrum of RGO showed that there was decrease in oxygen content and increase in carbon content of RGO when compared to GO. The optical analysis of the GO and RGO thin films gave a direct energy bandgap of $2.7 \mathrm{eV}$ and $2.2 \mathrm{eV}$ respectively. The value of sheet resistance of GO and RGO films was determined to be $22.9 \times 10^{6} \Omega / \mathrm{sq}$ and $4.95 \times$ $10^{6} \Omega$ /sq respectively.

\section{Keywords}

Graphite, Graphene, Graphene Oxide, Thin Films, Sheet Resistance

\footnotetext{
${ }^{*}$ Corresponding author.

How to cite this paper: Eluyemi, M.S., Eleruja, M.A., Adedeji, A.V., Olofinjana, B., Fasakin, O., Akinwunmi, O.O., Ilori, O.O., Famojuro, A.T., Ayinde, S.A. and Ajayi, E.O.B. (2016) Synthesis and Characterization of Graphene Oxide and Reduced Graphene Oxide Thin Films Deposited by Spray Pyrolysis Method. Graphene, 5, 143-154. 


\section{Introduction}

Graphene is a two-dimensional building block with honeycomb structure. It can be stacked as three dimensional graphite, rolled into one dimensional nanotubes, or folded into zero dimensional fullerenes [1] [2]. It is a nanoscale allotrope of carbon. Unlike graphite, electrons can only move between carbon atoms in the 2D lattice. The extra quantum confinement of the electrons due to the lack of a third dimension gives graphene various novel properties. For example, electrons interact with carbon atoms in the lattice to create a system that acts like a single mobile charge carrier. The carrier moves ballistically over the graphene surface which enables graphene sheets to conduct electricity [3] [4]. Other complex interactions between electrons and the hexagonal lattice make graphene transparent, flexible and strong [5]. These properties and others have attracted many researchers over the last half-decade to study graphene for a diverse array of uses [6] [7].

Graphite is a 3-dimensional carbon based material made up of millions of layers of graphene. By the oxidation of graphite using strong oxidizing agents, oxygenated functionalities are introduced into the graphite structure which not only expands the layer separation, but also makes the material hydrophilic. This property enables the graphite oxide to be exfoliated in water using sonication, ultimately producing single or few layer graphene, known as graphene oxide (GO). The main difference between graphite oxide and GO is, thus, the number of layers. In a GO dispersion, a few layer flakes and monolayer flakes can be found.

One of the advantages of GO is its easy dispersability in water and other organic solvents, as well as in different matrixes. This is due to the presence of the oxygen functionality groups. This remains a very important property in mixing the material with ceramic or polymer matrixes when trying to improve their electrical and mechanical properties [8] [9]. On the other hand, in terms of electrical conductivity, GO is often described as an electrical insulator, due to the disruption of its sp2 bonding networks. In order to recover the honeycomb hexagonal lattice and the electrical conductivity, the reduction of the graphene oxide has to be achieved. However, once most of the oxygen groups are removed, the reduced graphene oxide (RGO) obtained is more difficult to disperse due to its tendency to create aggregates.

Mass production of high quality graphene sheets at low cost is needed for commercial applications. Therefore, it is important to develop environmentally friendly methods for producing graphene nanosheets in large scale. Several methods have been developed to synthesize graphene sheets. This includes micromechanical exfoliation of graphite, chemical vapor deposition and epitaxial growth on silicon carbide. However, chemical reduction is regarded as the most promising approach for large volume synthesis. The chemical approach for producing GO is well suited for mass production because of its low-cost procedure. Furthermore, it can render a large variety of different variants of graphene with chemical modifications [10] [11].

In view of this, the interest of this study is to chemically synthesize GO and RGO through a modified Hummers' method, using natural flake graphite (NFG) as starting material. One of the advantages of this method is the absence of $\mathrm{NaNO}_{3}$, thus no generation of toxic gases such as $\mathrm{NO}_{2}, \mathrm{~N}_{2} \mathrm{O}_{4}$ in the reaction thereby making it more environmentally friendly. The use of this method is expected to be successful in terms of controlling the particle size, chemical composition and crystallite size in a simple and inexpensive way. Thin films of GO and RGO were also deposited on sodalime glass substrate using spray pyrolysis technique (SPT). The characterization of the prepared thin films samples is also reported.

\section{Experimental Method}

\subsection{Synthesis of GO}

In this study, a modified Hummers' method [12] was used to prepare GO. In this method, there is complete removal of sodium nitrate, increase in the amount of potassium permanganate and introduction of phosphoric acid [13]-[17]. $3.0 \mathrm{~g}$ of flake graphite (44 $\mu \mathrm{m}, 99.8 \%$ carbon, ash color, from Asbury Carbon, New Jersey, USA) was added to $120 \mathrm{ml}$ solution of sulphuric acid $\left(\mathrm{H}_{2} \mathrm{SO}_{4}\right)(98 \%$, $\mathrm{BDH})$ and phosphoric acid $\left(\mathrm{H}_{3} \mathrm{PO}_{4}\right)(98 \%$, $\mathrm{BDH})$ in a $500 \mathrm{ml}$ conical flask in ratio 9:1. The mixture was stirred vigorously and maintained at $100^{\circ} \mathrm{C}$ for 2 hours. The mixture was then allowed to cool to room temperature and kept below $10^{\circ} \mathrm{C}$ by placing the mixture in an ice bath. $15.0 \mathrm{~g}$ of potassium permanganate $\left(\mathrm{KMnO}_{4}\right)$ was added gradually over a period of one hour after which it was continuously stirred for another hour in order to obtain homogeneous mixture. It was then removed from ice-water bath, placed in a warm-water bath maintained at $45^{\circ} \mathrm{C}$ and stirred for an hour. After this, the mixture was allowed to cool to room temperature. It was observed that the color of the mixture changed to yellowish green. Subsequently, $250 \mathrm{ml}$ of distilled water was added to the mixture. The color of the mixture changed 
gradually from yellowish green to reddish brown as the reaction proceeds. In order to stop the reaction, $20 \mathrm{ml}$ of hydrogen peroxide (30\%, BDH) was added to the mixture until the color changed from reddish brown to dark brown. This shows that oxidized graphite has been synthesized. The obtained mixture was left overnight. The mixture was then centrifuged and re-dispersed in dilute hydrogen chloride. After this, it was repeatedly washed in distilled water and centrifuged three times. The mixture was tested with blue litmus paper each time it was washed in distilled water until the litmus paper color remained unchanged. This showed that the residual acid has been removed. Finally, the mixture was filtered, oven-dried and pulverized. The powder obtained was sieved so as to obtain even particle size.

\subsection{Reduction of GO}

The obtained GO was reduced using the method reported by Park et al. [18]. $300.0 \mathrm{~g}$ of GO was dispersed in $100 \mathrm{ml}$ of distilled water $(3 \mathrm{mg} / \mathrm{ml}) .0 .1 \mathrm{ml}$ of hydrazine monohydrate $\left(\mathrm{N}_{2} \mathrm{H}_{4}\right)$ was added to the mixture of GO and distilled water in a beaker. The mixture was placed in an oil bath, stirred and maintained at $80^{\circ} \mathrm{C}$ on a hot plate magnetic stirrer for one hour. After this duration, the color of the solution changed from dark brown to black which indicated that RGO has been obtained. The obtained RGO was washed thrice and filtered in order to remove residual $\mathrm{N}_{2} \mathrm{H}_{4}$ from the solution. The black filtrate was then oven-dried.

\subsection{Film Deposition}

In order to deposit GO and RGO thin films, $0.5 \mathrm{~g}$ each of GO powder and RGO powder was dispersed separately in $20 \mathrm{ml}$ of ethylene glycol (EG). EG was chosen because GO and RGO have high dispersability in it [19] [20]. The GO and RGO films were deposited using SPT. It is a technique which uses a liquid source for thin film coating of the desired compound on to preheated substrates [21]-[24]. The deposition temperature was $200^{\circ} \mathrm{C}$ while the distance between the nozzle and the substrate was $30 \mathrm{~cm}$. The temperature of the substrate was monitored using temperature probe connected to a digital multimeter. The carrier gas used was compressed air while the substrate used was sodalime glass. The sodalime glass showed a dark coloration after deposition of GO and RGO films which indicated the decomposition of the precursors.

\subsection{Characterization of Starting Materials and Thin Films}

Infrared spectrum of the NFG powder, GO powder and RGO powder was obtained using Fourier Transform Infrared spectrophotometer (FTIR, SHIMADZU IRTracer-100). The surface morphology and compositional analysis of the GO and RGO thin film materials were carried out using scanning electron microscope (SEM; 6510LA) with energy dispersive X-ray spectroscope (JOEL EDS System) facility attached to it. Optical absorbance of the GO and RGO thin films was obtained in order to study their optical property. This was done using Jenway UV-Visible spectrophotometer (Model 6405). All measurements were made at room temperature with incident photon wavelength ranging from 200 to $1100 \mathrm{~nm}$ at $5 \mathrm{~nm}$ interval. The electrical characterization of the films was done using Kethley four-point probe facility (Model 2400) with source voltage of $5 \mathrm{mV}$.

\section{Results and Discussion}

\subsection{FTIR Spectroscopy Analysis of GO and RGO}

FTIR analysis was used to identify functional groups present in the NFG, GO and RGO. Figures 1(a)-(c) show the FTIR spectrum of NFG, GO and RGO powders respectively. The NFG spectrum shows O-H broad peak at $3433.41 \mathrm{~cm}^{-1}$ resulting from intercalated water and $\mathrm{C}=\mathrm{C}$ at $1629.90 \mathrm{~cm}^{-1}$ which can be assigned to the skeletal vibrations of graphitic domains [25] [26]. Figure 1(b) confirmed the introduction of oxygen containing groups such as functional hydroxyl, epoxy and carboxylic groups after oxidation of NFG. The strong band at 1728.28 $\mathrm{cm}^{-1}$ is attributed to stretching vibration modes of $\mathrm{C}=\mathrm{O}$ in carboxylic acid and carbonyl groups. The peak at $1624.12 \mathrm{~cm}^{-1}$ is attributed to the skeletal vibrations of un-oxidized graphitic domains. The band at $1057.03 \mathrm{~cm}^{-1}$ is assigned to C-O (epoxy) groups while the band at $1224.84 \mathrm{~cm}^{-1}$ is usually attributed to C-OH stretching vibrations and $3433.41 \mathrm{~cm}^{-1}$ represents O-H stretching vibrations [9] [26]-[30].

After the reduction process using $\mathrm{N}_{2} \mathrm{H}_{4}$, there was drastic decrease in the bands associated with the oxygencontaining group. The broad band became sharp and finally, the peak at $1728.28 \mathrm{~cm}^{-1}$ for carboxyl $\mathrm{C}=\mathrm{O}$ entirely vanishes. Sharp peak at $1400.37 \mathrm{~cm}^{-1}$ corresponds to $\mathrm{C}=\mathrm{N}$ vibration while the one at $1637.62 \mathrm{~cm}^{-1} \mathrm{can} \mathrm{be}^{-1}$ 


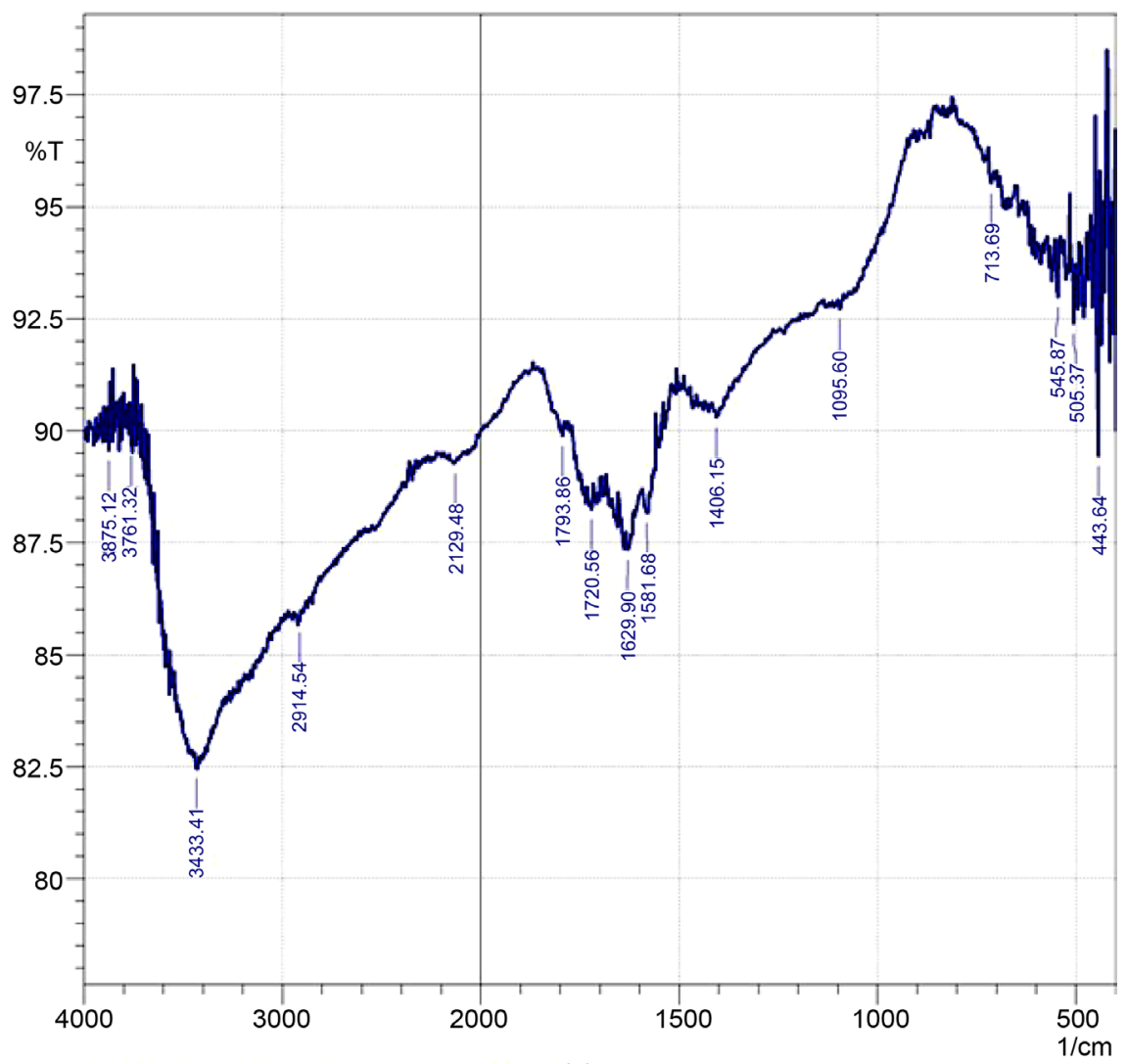

(a)

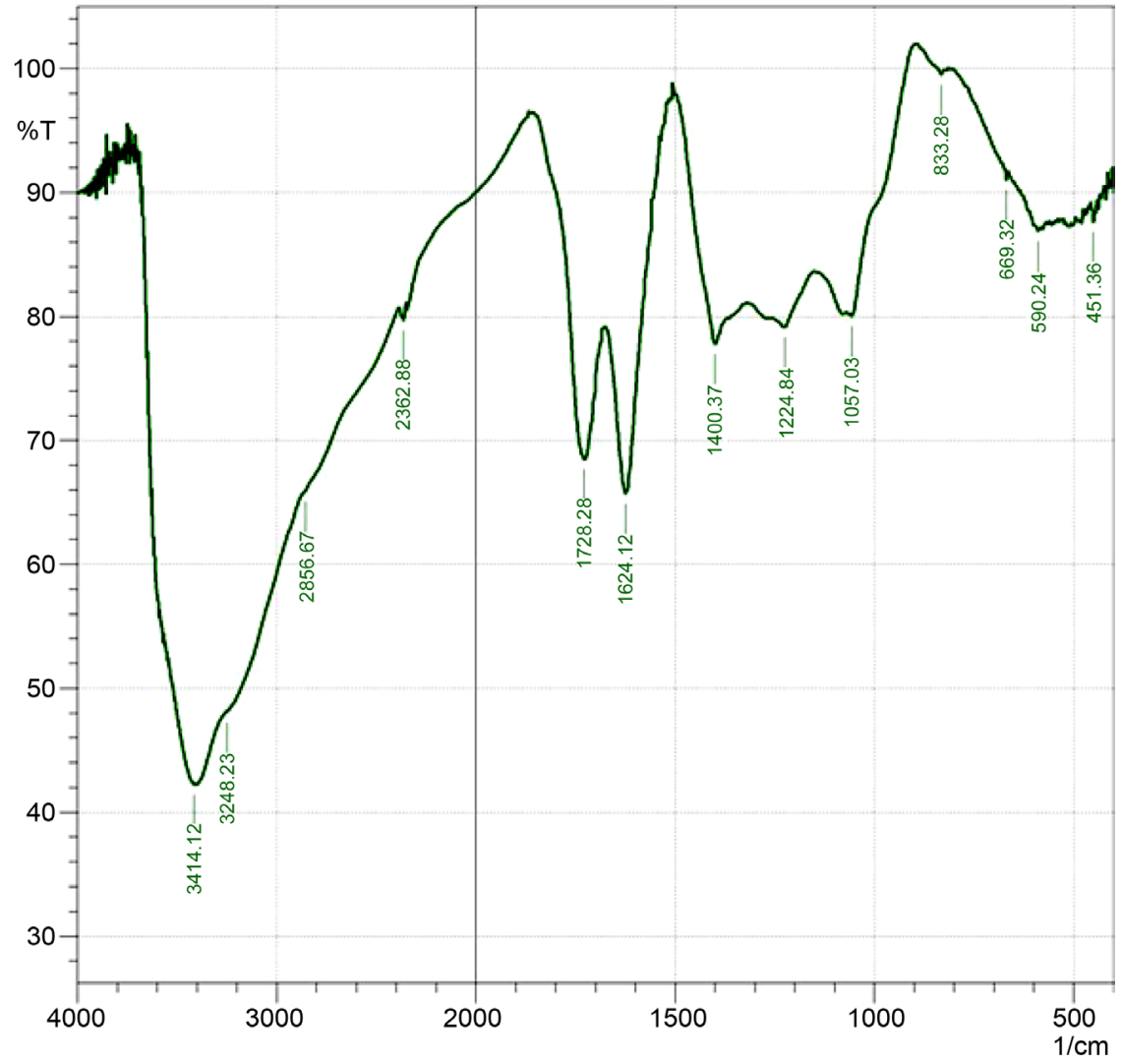

(b) 


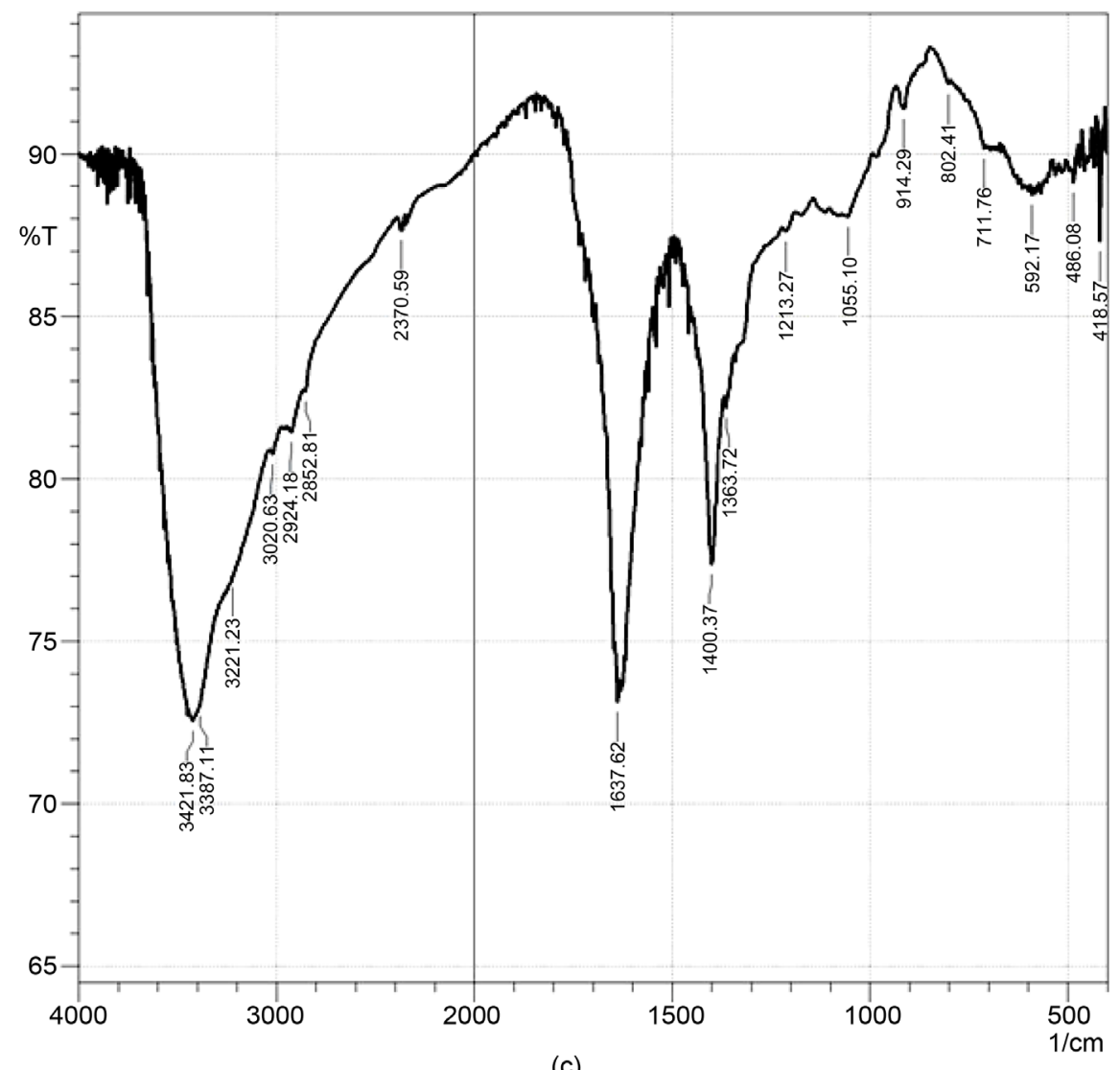

(c)

Figure 1. FTIR spectrum of (a) natural flake graphite powder (b) GO powder (c) RGO powder.

attributed to $\mathrm{C}=\mathrm{C}$ vibration [9] [25] [28] [29]. Amine group functionality was due to the hydrazine $\mathrm{N}_{2} \mathrm{H}_{4}$ used to reduce GO to RGO.

\subsection{Scanning Electron Microscope Analysis of GO and RGO Films}

The SEM micrographs of GO and RGO thin films are shown in Figure 2(a) and Figure 2(b). The micrograph of GO thin film has a porous sponge-like structure with the graphene sheet not well connected together. This is an indication that graphite has been exfoliated during oxidation process. This may be due to the distorted graphene sheets when oxygen and other functionality groups are attached to sheets of graphene to form GO [30]-[32]. The micrograph of RGO thin film showed evenly distributed and well connected graphene sheet, indicating that graphene sheets in RGO overlapped each other [33]. This observation showed that the normal plane of graphene has been restored while the oxygen and other functional groups attached to the sheets of graphene has decreased, after the reduction of GO to RGO. There was also a reduction in the particle size of GO from $100 \mathrm{~nm}$ to $10 \mathrm{~nm}$ in the case of RGO as detected by SEM using Image $\mathrm{J}$ software.

\subsection{Energy Dispersive X-Ray Spectroscopy Analysis of GO and RGO Films}

Figure 3(a) shows the EDX spectrum for the reference sodalime glass substrate. The spectrum showed peaks corresponding to $\mathrm{Si}, \mathrm{O}, \mathrm{Mg}, \mathrm{Na}, \mathrm{Al}$ and $\mathrm{Ca}$. Figure 3(b) shows the EDX spectrum of pelletized NFG with a peak that corresponds to carbon. The EDX spectrum of GO thin film is shown in Figure 3(c). The presence of carbon, oxygen, silicon, magnesium, sodium, phosphorus, sulphur and potassium can be seen. Phosphorus, sulphur and potassium were present as a result of $\mathrm{H}_{2} \mathrm{SO}_{4}, \mathrm{H}_{3} \mathrm{PO}_{4}$ and $\mathrm{KMnO}_{4}$ used as oxidizing agent, while silicon, magnesium and sodium are from the substrate. This is a confirmation that graphene oxide was actually formed. The EDX spectrum of RGO thin film shown in Figure 3(d) also confirmed the presence of carbon, oxygen, silicon, and sodium. However, it can be seen that phosphorus, sulphur and potassium were absent. There is also a 


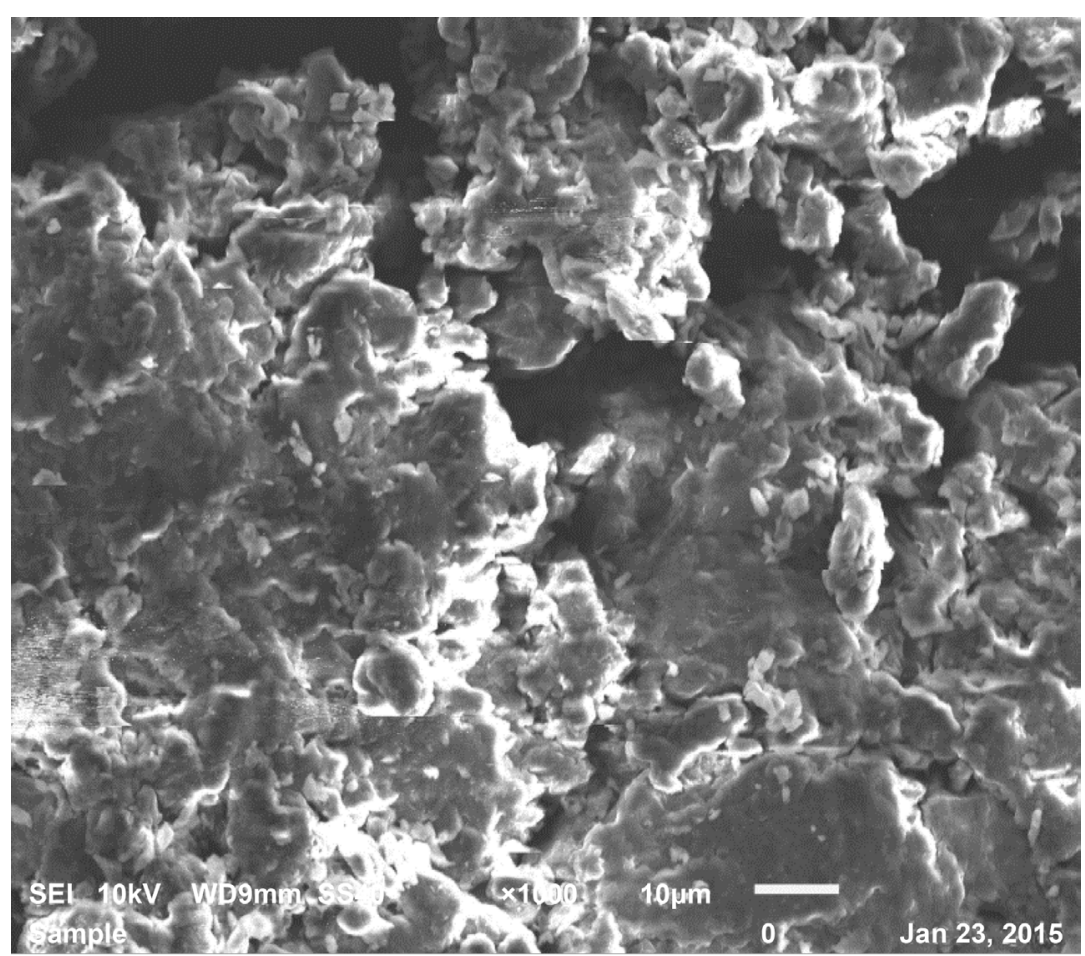

(a)

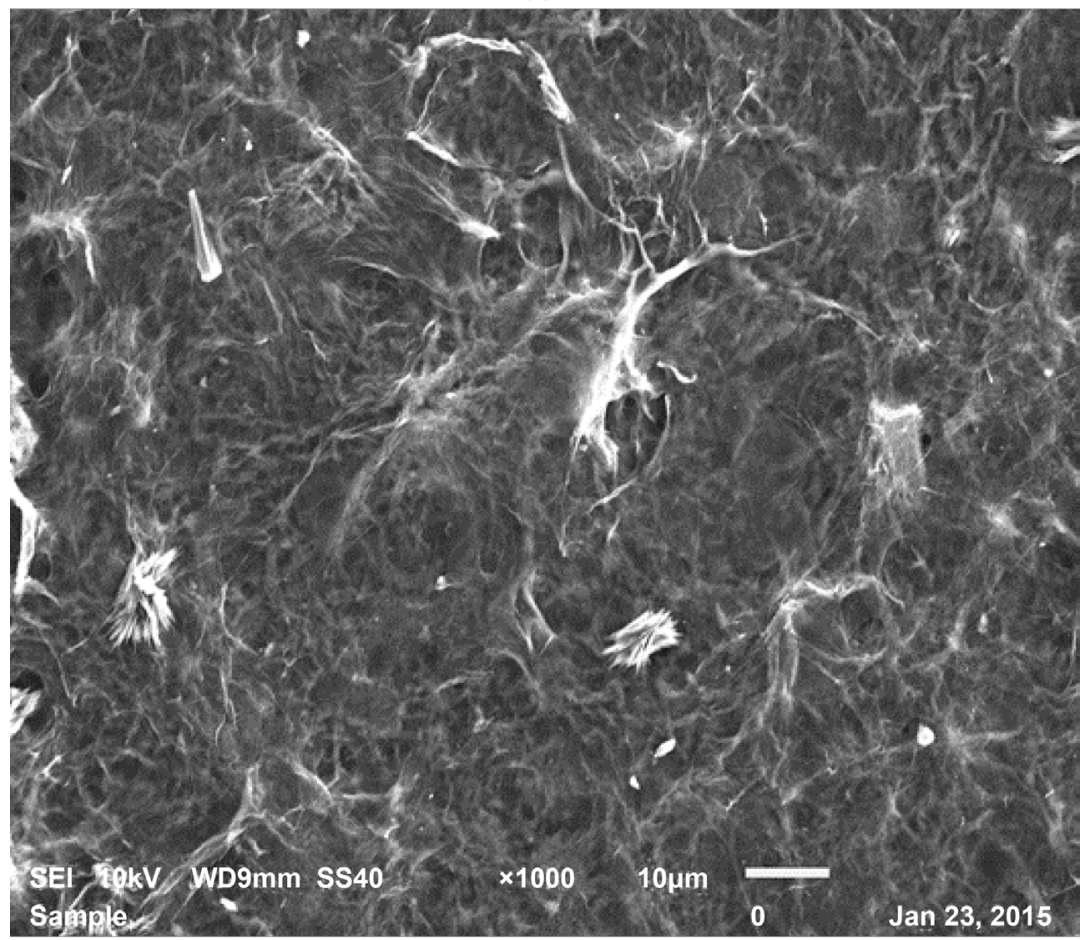

(b)

Figure 2. SEM micrograph of (a) GO (b) RGO thin film.

reduction in percentage of oxygen and increase in percentage of carbon when compared with that of GO. The mass ratio of $\mathrm{C}: \mathrm{O}$ in GO and RGO films was estimated to 5:8 and 12:5 respectively. The atom ratio of C:O in GO and RGO films was also estimated to be 4:5 (0.8) and 3:1 (3.0) respectively. This result showed that $\mathrm{N}_{2} \mathrm{H}_{4}$ reduced GO to RGO to a certain extent. 

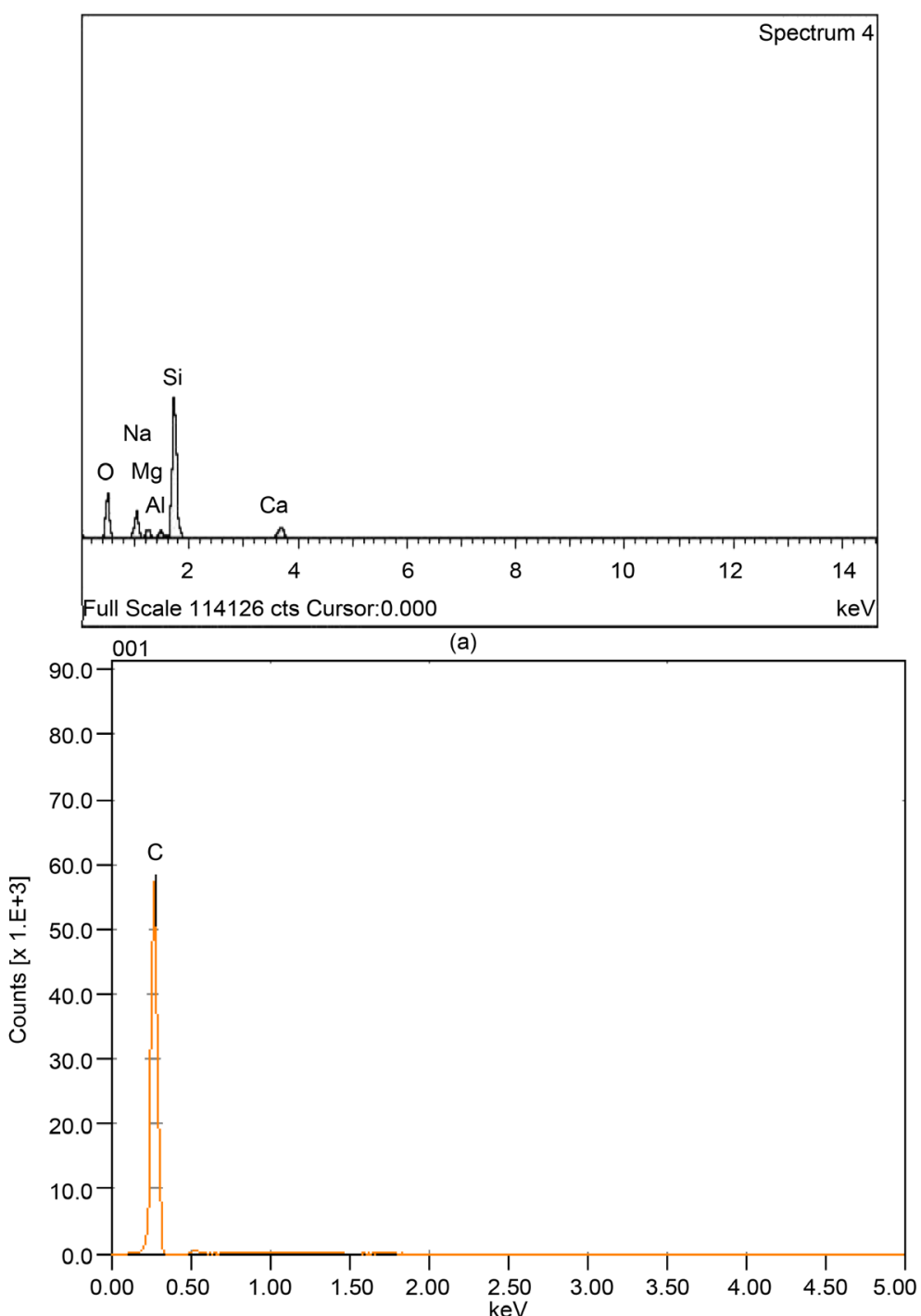

001

(b)

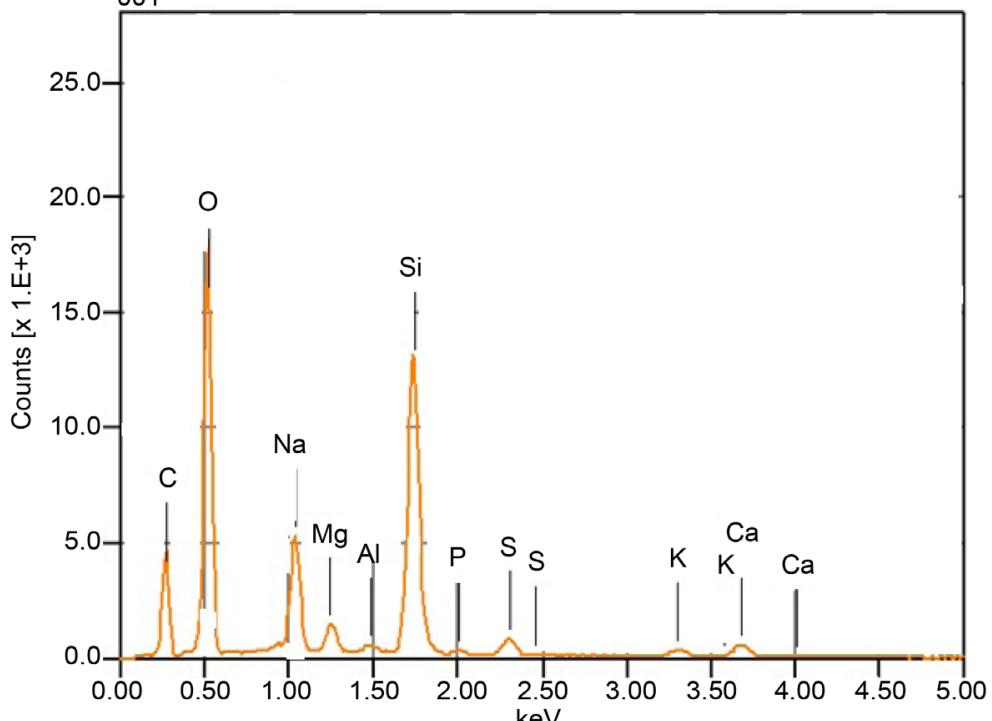

(c) 


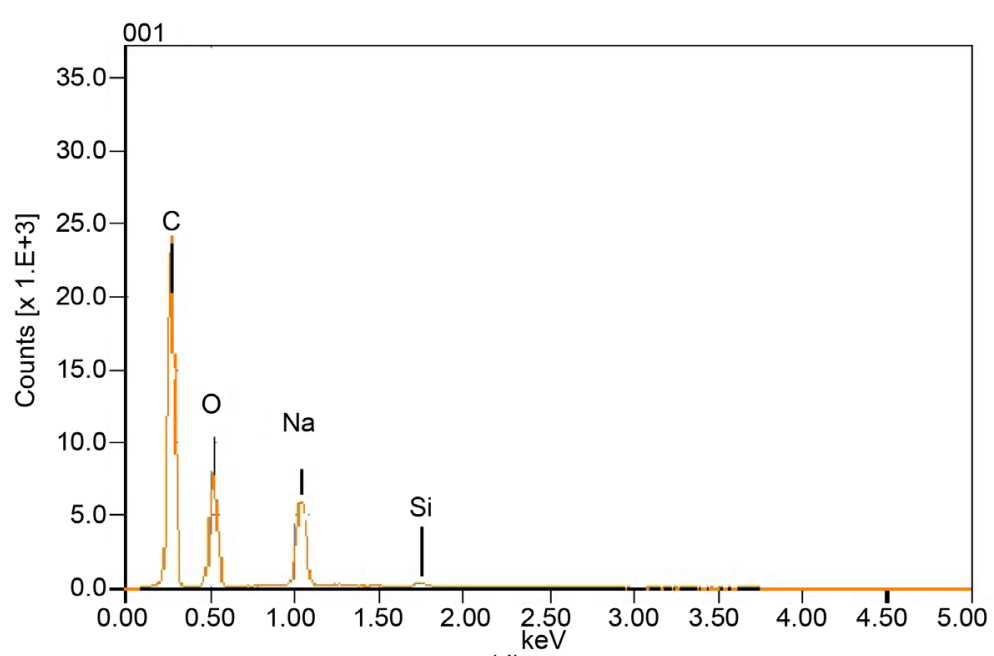

(d)

Figure 3. EDX spectrum of (a) sodalime glass substrate (b) pelletized NFG (c) GO thin film on sodalime glass substrate (d) RGO thin film on sodalime glass substrate.

\subsection{Optical Characterization of GO and RGO Thin Films}

Optical characterization constitutes the most direct and perhaps the simplest approach for probing the band structure of the deposited film. The UV-Visible spectrum of the thin film is shown in Figure 4(a). The UVVisible spectrum is a plot of the absorbance, $A$ as a function of wavelength, $\lambda$. It can be seen that GO has higher absorbance when compared to that of RGO. The absorbance peak of GO dispersion is in the range of 250 to 300 $\mathrm{nm}$. A major peak also occurs at approximately $260 \mathrm{~nm}$. Since absorbance depends on atomic number and density of material, this peak may be due to the attachment of functional group to layers of graphene in GO. The absorbance peak of RGO is around $210 \mathrm{~nm}$ [27]. The decrease in the absorbance peak of RGO when compared with that of GO is due to decrease in the amount of functional group after reduction process [34]. The absorption coefficient $\alpha$ is given by

$$
\alpha=\frac{1}{d} \ln \frac{1}{T}
$$

where, $d$ is the thickness of the film, $T=10^{-A}$ is the transmittance. The dependence of absorption coefficient on photon energy can be obtained for optical transition processes using the time-dependent perturbation theory. The optical absorption at high values of absorption coefficient follows a power law of the general form

$$
\alpha=B\left(h v-E_{g}\right)^{n}
$$

where $h=$ Planck's constant, $v=$ frequency, $E_{g}=$ energy gap and $B=$ constant of proportionality. The exponent, $n$, which characterizes the transition process can be taken as $1 / 2$. From Equation (1), square of the absorption coefficient, $\alpha^{2}$ was calculated. Figure 4(b) shows the plot of $\alpha^{2}$ against the energy of the photon for the GO and RGO thin films. The extrapolation of linear part of the plot to the energy axis gives the direct bandgap energy of the GO and RGO thin films to be $2.7 \mathrm{eV}$ and $2.2 \mathrm{eV}$ respectively. This implies that reduction of GO to RGO lower the bandgap of GO which can lead to increase in the conductivity of RGO [27].

\subsection{Electrical Characterization of the Films}

The electrical characterization of GO and RGO films was carried out using Keithley (Model 2400) four-point probes. The values of current and equivalent voltage were taken. The average current generated was $4.946 \times$ $10^{-9} \mathrm{~A}$ and $45.6903 \times 10^{-9} \mathrm{~A}$ for GO and RGO thin film respectively, while the average voltage determined was $25.00 \mathrm{mV}$ and $50.00 \mathrm{mV}$ for GO and RGO thin film respectively. Sheet resistance $R_{s}$ of GO and RGO thin film was determined using the equation,

$$
R_{s}=K \frac{V}{I}
$$




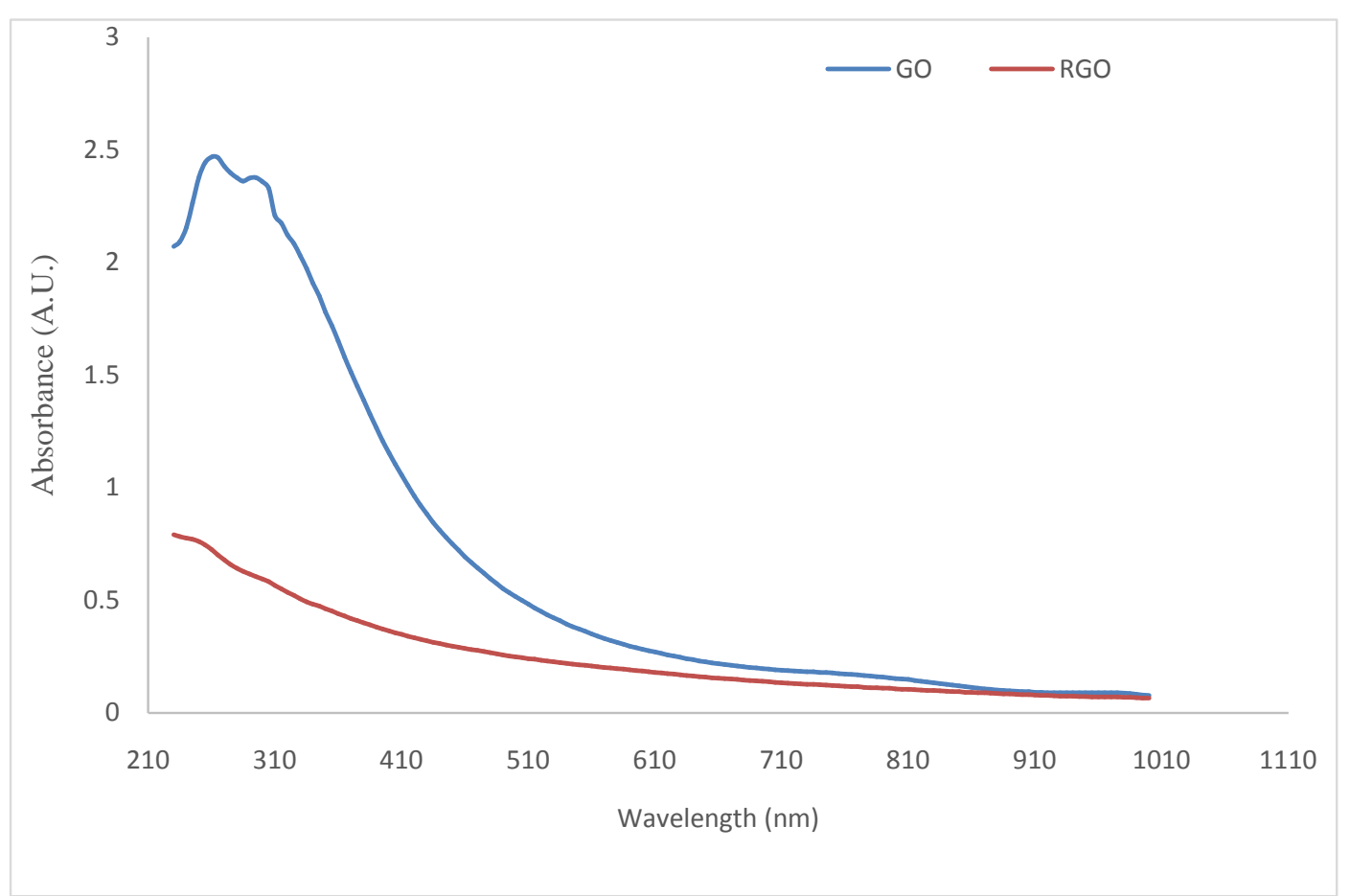

(a)

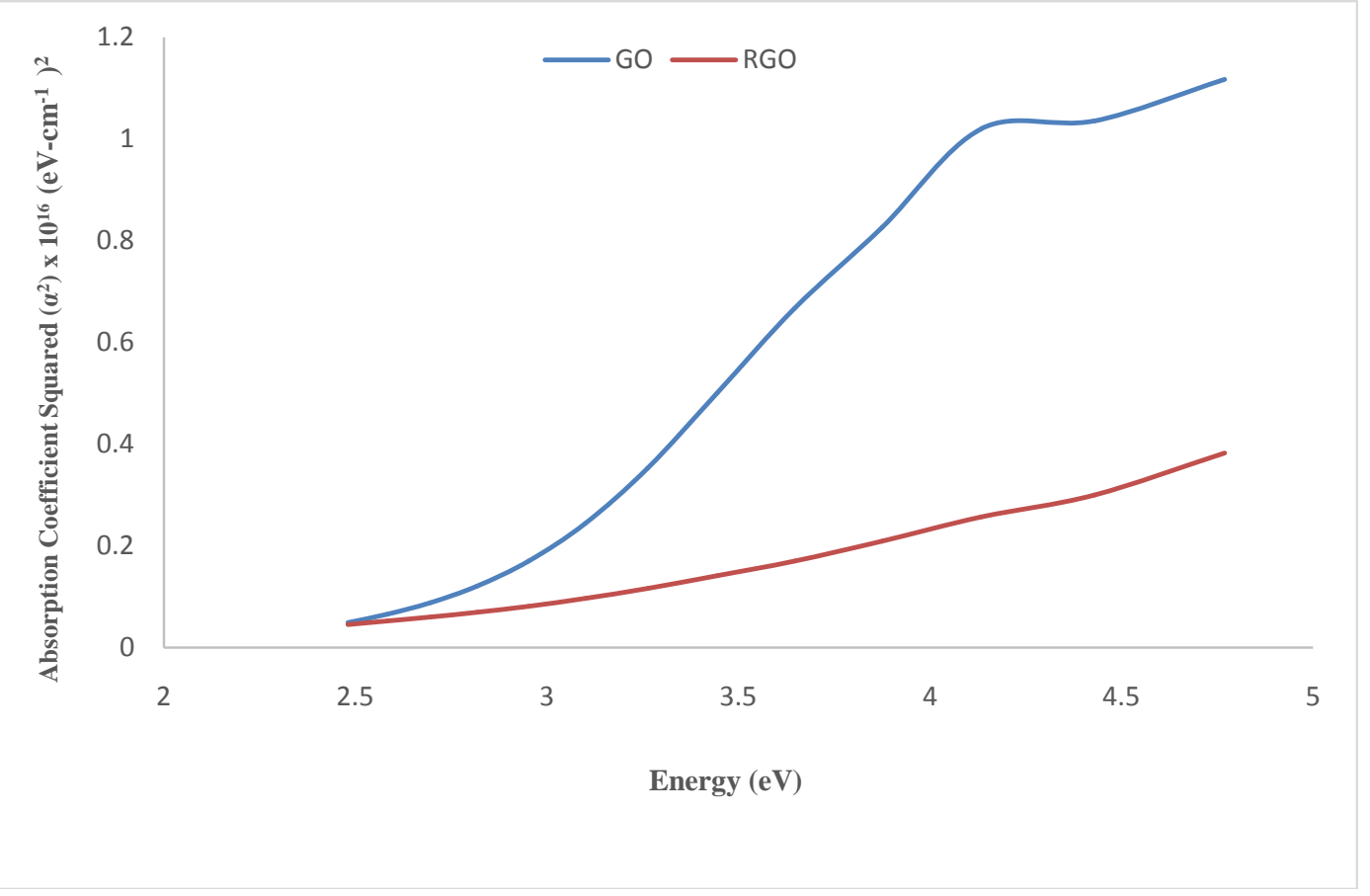

(b)

Figure 4. (a) UV-visible Spectra of the GO and RGO thin films (b) The Graph of $\alpha^{2}$ against Energy for the GO and RGO thin films.

where $K$ is a constant that depends on the configuration and spacing of the probes. In this case,

$$
K=\frac{\pi}{\operatorname{In} 2}=4.532 \text {. }
$$


The sheet resistance of GO and RGO thin film was calculated to be $22.9 \times 10^{6} \Omega / \mathrm{sq}$ and $4.95 \times 10^{6} \Omega / \mathrm{sq}$ respectively. The result obtained is of the same order of magnitude with that reported in literature [16] [35]. The resistivity of GO and RGO thin films was calculated in terms of average sheet resistance using equation,

$$
\rho=R_{s} t
$$

where $t$ is the thickness of the film which was estimated, using color code chart [36]. The resistivity, $\rho$ of GO and RGO thin film was estimated to be $0.6183 \Omega-\mathrm{m}$ and $0.13365 \Omega-\mathrm{m}$. The reduction in resistivity indicated that GO has been drastically reduced to RGO.

\section{Conclusions}

GO was synthesized from NFG powder and chemically reduced using $\mathrm{N}_{2} \mathrm{H}_{4}$ to obtain reduced RGO. Thin films of GO and RGO were also deposited on sodalime glass substrate using SPT. The functional groups present in the synthesized GO and RGO were characterized using FTIR spectroscopy. Morphological property, chemical composition, optical and electrical properties of the deposited thin films were also investigated.

FTIR spectrum of GO showed the introduction of oxygen-containing functional group which confirmed the oxidation of the NGF to form GO. The drastic reduction of oxygen-containing functional group in the FTIR spectrum of RGO also confirmed the reduction of GO to RGO. SEM micrograph of GO thin film showed a porous sponge-like structure with the graphene sheet not well connected together which is an indication that graphite has been exfoliated to GO. The micrograph of RGO thin film showed evenly distributed and well connected graphene which is an indication that the normal plane of graphene has been restored while the oxygen and other functionality groups attached to sheets of graphene have decreased after the reduction of GO to RGO. The EDX spectrum of RGO showed that there was decrease in oxygen content and increase in carbon content when compared to that of GO. The optical behavior showed that the absorption peak of the GO and RGO thin films were within visible range which corresponding to a direct energy bandgap of $2.7 \mathrm{eV}$ and $2.2 \mathrm{eV}$ for GO and RGO thin films respectively. The values of sheet resistance and resistivity of GO and RGO films were determined to be $22.9 \times 10^{6} \Omega /$ sq and $0.6183 \Omega$-m; $4.95 \times 10^{6} \Omega /$ sq and $0.13365 \Omega$-m respectively.

These results indicated low oxygen content, evenly distributed well connected graphene structure, reduction in bandgap and decrease in resistivity of RGO thin film when compared with GO thin film. Hence, the method adopted in this work is suitable for preparing GO and RGO precursors for the deposition of their respective thin films using SPT. The results of the thin films characterizations showed that the use of this method is a promising technique for the development of GO and RGO thin films for use in various technological applications such as energy storage devices and solar cell applications.

\section{Acknowledgements}

The synthesis of the samples was carried out in the Chemistry Laboratory of Centre for Energy Research and Development, Obafemi Awolowo University, Ile-Ife Nigeria. Our Sincere appreciation goes to Dr. V.T. Albert of Asbury Graphite Mills, New Jersey, USA for the supply of the Natural Flake Graphite for this research.

\section{References}

[1] De, D., Chakraborty, M., Majumdar, S. and Giri, S. (2014) Bandgap Engineering through Nanocrystalline Magnetic Alloy Grafting on Reduced Graphene Oxide. Physical Chemistry Chemical Physics, 16, 19661-19667. http://dx.doi.org/10.1039/C4CP02259A

[2] Garg, B., Bisht, T. and Ling, Y.C. (2014) Graphene-Based Nanomaterials as Heterogeneous Acid Catalysts: A Comprehensive Perspective. Molecules, 19, 14582-14614. http://dx.doi.org/10.3390/molecules190914582

[3] Geim, A.K. and Novoselov, K.S. (2007) The Rise of Graphene. Nature Materials, 6, 183-191. http://dx.doi.org/10.1038/nmat1849

[4] Gethers, M.L., Thomas, J.C., Jiang, S., Weiss, N.O., Duan, X., Goddardlll, W.A. and Weiss, P.S. (2015) Holey Graphene as a Weed Barrier for Molecules. ACS Nano, 9, 10909-10915. http://dx.doi.org/10.1021/acsnano.5b03936

[5] Bunch, J.S., Verbridge, S.S., Alden, J.S., van der Zande, A.M., Parpia, J.M., Craighead, H.G. and McEuen, P.L. (2008) Impermeable Atomic Membranes from Graphene Sheets. Nano Letters, 8, 2458-2462. http://dx.doi.org/10.1021/nl801457b

[6] Chen, T., Xia, Y., Jia, Z., Liu, Z. and Zhang, H. (2014) Synthesis, Characterization and Tribological Behavior of Oleic 
Acid Capped Graphene Oxide. Journal of Nanomaterials, 2014, Article ID: 654145. http://dx.doi.org/10.1155/2014/654145

[7] Wu, Z.S., Sun, Y., Tan, Y.Z., Yang, S., Feng, X. and Mullen, K. (2012) Three-Dimensional Graphene-Based Macroand Mesoporous Frameworks for High-Performance Electrochemical Capacitive Energy Storage. Journal of the American Chemical Society, 134, 19532-19535. http://dx.doi.org/10.1021/ja308676h

[8] Cao, Y., Feng, J. and Wu, P. (2010) Preparation and Organically Dispersible Graphene Nanosheet Powders through a Lyophilization Method and Their Poly(lactic acid) Composites. Carbon, 48, 3834-3839. http://dx.doi.org/10.1016/j.carbon.2010.06.048

[9] Yoo, D., Kim, J. and Kim, J.H. (2014) Direct Synthesis of Highly Conductive PEDOT: PSS/Graphene Composites and Their Applications in Energy Harvesting Systems. Nano Research, 7, 717-730. http://dx.doi.org/10.1007/s12274-014-0433-z

[10] Wan, W., Zhao, Z., Hu, H. and Qiu, J.S. (2013) Highly Controllable and Green Reduction of Graphene Oxide to Flexible Graphene Film with High Strength. Materials Research Bulletin, 48, 4797-4803. http://dx.doi.org/10.1016/j.materresbull.2013.08.031

[11] Alam, S.N., Kumar, L. and Sharma, N. (2015) Development of Cu-Exfoliated Graphite Nanoplatelets (xGnP) Metal Matrix Composite by Powder Metallurgy Route. Graphene, 4, 91-111. http://dx.doi.org/10.4236/graphene.2015.44010

[12] Hummers Jr., W.S. and Offeman, R.E. (1958) Preparation of Graphitic Oxide. Journal of Chemical Society, 80, 13391339. http://dx.doi.org/10.1021/ja01539a017

[13] Liu, W.R., Kuo, S.L., Lin, C.Y., Chiu, Y.C., Su, C.Y., Wu, H.C. and Hsieh, C.T. (2011) Characterization and Electrochemical Behavior of Graphene-Based Anode for Li-ion Batteries. The Open Materials Science Journal, 5, $236-242$. http://dx.doi.org/10.2174/1874088X01105010236

[14] Dinesh, M.M., Saminathan, K., Selvam, M., Srither, S.R., Rajendran, V. and Kaler, V.I.S. (2015) Water Soluble Graphene as Electrolyte Additive in Magnesium-Air Battery System. Journal of Power Source, 276, 32-38. http://dx.doi.org/10.1016/j.jpowsour.2014.11.079

[15] Srisvastava, P.K., Yadav, P. and Ghosh, S. (2015) Dielectric Environment as a Factor to Enhance the Production Yield of Solvent Exfoliated Graphene. RSC Advances, 5, 64395-64399. http://dx.doi.org/10.1039/C5RA12464F

[16] Kim, F., Cote, L.J. and Huang, J. (2010) Graphene Oxide: Surface Activity and Two Dimensional Assembly. Advanced Materials, 22, 1954-1958. http://dx.doi.org/10.1002/adma.200903932

[17] Zhu, Y., Murali, S., Cai, W., Li, X., Suk, J.W., Potts, J.R. and Ruoff, R.S. (2010) Graphene and Graphene Oxide: Synthesis, Properties, and Applications. Advanced Materials, 22, 3906-3924. http://dx.doi.org/10.1002/adma.201001068

[18] Park, S., An, J., Potts, J.R., Velamakanni, A., Murali, S. and Ruoff, R. (2011) Hydrazine-Reduction of Graphite and Graphene Oxide. Carbon, 49, 3019-3023. http://dx.doi.org/10.1016/j.carbon.2011.02.071

[19] Choi, E.Y., Han, T.H., Hong, J., Kim, J.E., Lee, S.H., Kim, H.W. and Kim, S.O. (2009) Noncovalent Functionalization of Graphene with End-Functional Polymers. Journal of Materials Chemistry, 20, 1907-1912. http://dx.doi.org/10.1039/b919074k

[20] Dreyer, D.R., Park, S., Bielawski, C.W. and Ruoff, R.S. (2010) The Chemistry of Graphene Oxide. Chemical Society Review, 39, 228-240. http://dx.doi.org/10.1039/B917103G

[21] Filipovic, L., Selberherr, S., Mutinati, G.C., Brunet, E., Steinhauer, S., Köck, A., Teva, J., Kraft, J., Siegart, J. and Shrank, F. (2014) Methods of Simulating Thin Film Deposition Using Spray Pyrolysis Techniques. Microelectronic Engineering, 117, 57-66. http://dx.doi.org/10.1016/j.mee.2013.12.025

[22] Lean, I.M. and Reinhard, G. (1972) Handbook of Thin Film Technology. McGraw-Hill Book, New York.

[23] Perednis, D. and Gauckler, L.J. (2005) Thin Film Deposition Using Spray Pyrolysis. Journal of Electroceramic, 14, 103-111. http://dx.doi.org/10.1007/s10832-005-0870-X

[24] Guild, C., Biswas, S., Meng, Y., Jafari, T., Gaffney, A.M. and Suib, S.L. (2014) Perspectives of Spray Pyrolysis for Facile Synthesis of Catalysts and Thin Films: An Introduction and Summary of Recent Directions. Catalysis Today, 238, 87-94. http://dx.doi.org/10.1016/j.cattod.2014.03.056

[25] Coates, J. (2000) Interpretation of Infrared Spectra: A Practical Approach. In: Meyers, R.A., Ed., Encyclopedia of Analytical Chemistry, John Wiley \& Sons Ltd., Chichester, 10881-10882.

[26] Yang, B., Liu, Z., Guo, Z., Zhang, W., Wan, M., Qin, X. and Zhong, H. (2014) In Situ Green Synthesis of Silver-Graphene Oxide Nanocomposites by Using Tryptophan as a Reducing and Stabilizing Agent and Their Application in SERS. Applied Surface Science, 316, 22-27. http://dx.doi.org/10.1016/j.apsusc.2014.07.084

[27] Nyangiwe, N.N., Khenfouch, M., Thema, F.T., Nukwa, K., Kotsedi, L. and Maaza, M. (2015) Free-Green Synthesis and Dynamics of Reduced Graphene Sheets via Sun Light Irradiation. Graphene, 4, 54-61. http://dx.doi.org/10.4236/graphene.2015.43006 
[28] Somanathan, T., Prasad, K., Ostrikov, K.K., Saravanan, A. and Krishna, V.M. (2015) Graphene Oxide Synthesis from Agro Waste. Nanomaterials, 5, 826-834. http://dx.doi.org/10.3390/nano5020826

[29] Wojtoniszak, M.E. (2012) Controlled Oxidation of Graphite to Graphene Oxide with Novel Oxidants in a Bulk Scale. Journal of Nanoparticle Research, 14, 1248-1255. http://dx.doi.org/10.1007/s11051-012-1248-Z

[30] Liu, F., Cao, Y., Yi, M., Xie, L., Huang, W., Tang, N., Zhong, W. and Du, Y. (2013) Thermostability, Photoluminescence, and Electrical Properties of Reduced Graphene Oxide-Carbon Nanotube Hybrid Materials. Crystals, 3, 28-37. http://dx.doi.org/10.3390/cryst3010028

[31] Geng, J., Liu, L., Yang, S.B., Youn, S.C., Kim, D.W., Lee, J.S., Choi, J.K. and Jung, H.T. (2010) A Simple Approach for Preparing Transparent Conducting Graphene Films Using the Controlled Chemical Reduction of Exfoliated Graphene Oxide in an Aqueous Suspension. The Journal of Physical Chemistry C, 114, 14433-14440. http://dx.doi.org/10.1021/jp105029m

[32] Eda, G. and Chhowlla, M. (2010) Chemically Derived Graphene Oxide: Towards Large Area Thin-Film Electronics and Optoelectronics. Advanced Materials, 22, 2392-2415. http://dx.doi.org/10.1002/adma.200903689

[33] Gudarzi, M.M. and Sharif, F. (2012) Enhancement of Dispersion and Bonding of Graphene-Polymer through Wet Transfer of Funtionalized Graphene Oxide. eXPRESS Polymer Letters, 6, 1017-1031. http://dx.doi.org/10.3144/expresspolymlett.2012.107

[34] Danilov, M.O., Slobodyanyuk, I.A., Rusetskii, I.A. and Kolbasov, G.Y. (2013) Reduced Graphene Oxide: A Promising Electrode Material for Oxygen Electrodes. Journal of Nanostructure in Chemistry, 3, 49-55. http://dx.doi.org/10.1186/2193-8865-3-49

[35] Becerrilt, H.A., Mao, J., Liu, Z., Stoltenberg, R.M., Bao, Z. and Chen, Y. (2008) Evaluation of Solution-Processed Reduced Graphene Oxide Films as Transparent Conductor. ACS Nano, 2, 463-470. http://dx.doi.org/10.1021/nn700375n

[36] Schroder, D.K. (2006) Semiconductor Material and Device Characterization. John Wiley \& Sons Inc., New York.

\section{Submit or recommend next manuscript to SCIRP and we will provide best service for you:}

Accepting pre-submission inquiries through Email, Facebook, LinkedIn, Twitter, etc.

A wide selection of journals (inclusive of 9 subjects, more than 200 journals)

Providing 24-hour high-quality service

User-friendly online submission system

Fair and swift peer-review system

Efficient typesetting and proofreading procedure

Display of the result of downloads and visits, as well as the number of cited articles

Maximum dissemination of your research work

Submit your manuscript at: http://papersubmission.scirp.org/ 